\title{
SUBCANONICAL POINTS ON PROJECTIVE CURVES AND TRIPLY PERIODIC MINIMAL SURFACES IN THE EUCLIDEAN SPACE
}

\author{
FRANCESCO BASTIANELLI AND GIAN PIETRO PIROLA
}

\begin{abstract}
A point $p \in C$ on a smooth complex projective curve of genus $g \geq 3$ is subcanonical if the divisor $(2 g-2) p$ is canonical. The subcanonical locus $\mathcal{G}_{g} \subset \mathcal{M}_{g, 1}$ described by pairs $(C, p)$ as above has dimension $2 g-1$ and consists of three irreducible components. Apart from the hyperelliptic component $\mathcal{G}_{g}^{\text {hyp }}$, the other components $\mathcal{G}_{g}^{\text {odd }}$ and $\mathcal{G}_{g}^{\text {even }}$ depend on the parity of $h^{0}(C,(g-1) p)$, and their general points satisfy $h^{0}(C,(g-1) p)=1$ and 2 , respectively. In this paper, we study the subloci $\mathcal{G}_{g}^{r}$ of pairs $(C, p)$ in $\mathcal{G}_{g}$ such that $h^{0}(C,(g-1) p) \geq r+1$ and $h^{0}(C,(g-1) p) \equiv r+1(\bmod 2)$. In particular, we provide a lower bound on their dimension, and we prove its sharpness for $r \leq 3$. As an application, we further give an existence result for triply periodic minimal surfaces immersed in the 3-dimensional Euclidean space, completing a previous result of the second author.
\end{abstract}

\section{INTRODUCTION}

The study of subcanonical points involves two very classical topics in the theory of algebraic curves: Weierstrass points and theta-characteristics. Let $C$ be a smooth complex projective curve of genus $g$. A point $p \in C$ is a subcanonical point if the line bundle $\mathcal{O}_{C}((2 g-2) p)$ is isomorphic to the canonical bundle $\omega_{C}$. In particular, such a $p \in C$ is a Weierstrass point, that is $h^{0}(C, g p)=$ $\operatorname{dim} H^{0}(C, g p)>1$, and the line bundle $\mathcal{L}=\mathcal{O}_{C}((g-1) p)$ is a theta-characteristic, as $\mathcal{L}^{\otimes 2} \cong \omega_{C}$.

Let $\mathcal{M}_{g, 1}$ denote the moduli space of pointed curves of genus $g$, and let $\mathcal{G}_{g} \subset \mathcal{M}_{g, 1}$ be the subcanonical locus, which is described by pairs $(C, p)$ such that $p$ is a subcanonical point of $C$. It follows from the work of Kontsevich and Zorich [14] that for $g>3, \mathcal{G}_{g}$ consists of three irreducible components $\mathcal{G}_{g}^{\text {hyp }}, \mathcal{G}_{g}^{\text {odd }}$ and $\mathcal{G}_{g}^{\text {even }}$ having dimension $2 g-1$. The locus $\mathcal{G}_{g}^{\text {hyp }}$ parameterizes hyperelliptic curves with a Weierstrass point, whereas the components $\mathcal{G}_{g}^{\text {odd }}$ and $\mathcal{G}_{g}^{\text {even }}$ correspond to non-hyperelliptic curves such that $h^{0}(C,(g-1) p)$ is odd and even, respectively.

Every pointed hyperelliptic curve $(C, p)$ lying on the locus $\mathcal{G}_{g}^{\text {hyp }}$ satisfies $h^{0}(C, n p)=\left\lfloor\frac{n+2}{2}\right\rfloor$ for any $0 \leq n \leq 2 g-2$ (see e.g. [11, p. 274]), whereas the sequence of integers $h^{0}(C, n p)$ for pointed curves in $\mathcal{G}_{g}^{\text {odd }}$ and $\mathcal{G}_{g}^{\text {even }}$ has been described only in the general case. In particular, Bullock showed that the general point $[C, p] \in \mathcal{G}_{g}^{\text {odd }}$ satisfies $h^{0}(C,(g-1) p)=1$, whereas the general $[C, p] \in \mathcal{G}_{g}^{\text {even }}$ is such that $h^{0}(C,(g-1) p)=2$ and the linear series $|(g-1) p|$ is base-point-free (see [4]).

Then it is interesting to investigate dimension and irreducibility of subsets of $\mathcal{G}_{g}^{\text {odd }}$ and $\mathcal{G}_{g}^{\text {even }}$ consisting of pointed curves whose associated theta-characteristic has higher number of sections. In this paper we discuss such a problem, and we focus on the subloci of $\mathcal{M}_{g, 1}$ defined as

$$
\mathcal{G}_{g}^{r}:=\left\{\begin{array}{l|l}
{[C, p] \in \mathcal{G}_{g}} & \begin{array}{l}
C \text { is non-hyperelliptic }, h^{0}(C,(g-1) p) \geq r+1 \\
\text { and } h^{0}(C,(g-1) p) \equiv r+1(\bmod 2)
\end{array}
\end{array}\right\} .
$$

This work was partially supported by INdAM (GNSAGA); PRIN 2012 "Moduli, strutture geometriche e loro applicazioni"; FAR 2013 (PV) "Varietà algebriche, calcolo algebrico, grafi orientati e topologici"; FIRB 2012 "Spazi di moduli e applicazioni". 
In particular, we provide a general bound on the dimension of $\mathcal{G}_{g}^{r}$, and we prove its sharpness for $r \leq 3$. Furthermore, as an application of the results obtained in the case $r=2$, we complete an existence result included in [20] for triply periodic minimal Riemann surfaces immersed in the 3-dimensional Euclidean space.

It is clear from the definition that $\mathcal{G}_{g}^{0}=\mathcal{G}_{g}^{\text {odd }}, \mathcal{G}_{g}^{1}=\mathcal{G}_{g}^{\text {even }}$ and the locus $\mathcal{G}_{g}^{r}$ is contained in $\mathcal{G}_{g}^{\text {odd }}$ (resp. $\mathcal{G}_{g}^{\text {even }}$ ) when $r+1$ is odd (resp. even). We note further that early bounds on the dimension of $\mathcal{G}_{g}^{r}$ may be obtained from the classical theory of Weierstrass points. However they turn out to fail being sharp as $r \geq 1$ (see Remark 2.1).

On the other hand, we interpret subcanonical points in terms of theta-characteristics, and we consider the loci $\mathcal{M}_{g}^{r} \subset \mathcal{M}_{g}$ of curves possessing a theta-characteristic with at least $r+1$ global sections and the same parity as $r+1$. Then, using Harris' bound on the codimension of $\mathcal{M}_{g}^{r}$ in $\mathcal{M}_{g}$ (see [12]), we achieve the following.

Theorem 1.1. Let $r \geq 0$. Then either $\mathcal{G}_{g}^{r}$ is empty or any irreducible component $\mathcal{Z}$ of $\mathcal{G}_{g}^{r}$ has dimension

$$
\operatorname{dim} \mathcal{Z} \geq 2 g-\frac{r(r-1)}{2}-1
$$

It follows from the description of $\mathcal{G}_{g}^{\text {odd }}$ and $\mathcal{G}_{g}^{\text {even }}$ that the latter bound is actually an equality both when $r=0,1$ with $g \geq 4$, and when $r=2$ as long as $\mathcal{G}_{g}^{2}$ is non-empty. In this direction, we prove the following.

Theorem 1.2. For any $g \geq 6$, the locus $\mathcal{G}_{g}^{2}$ is non-empty and every irreducible component $\mathcal{G}_{g}^{2}$ has dimension $2 g-2$.

In particular, we construct a non-empty component of $\mathcal{G}_{g}^{2}$ whose general point $[C, p]$ is such that $h^{0}(C,(g-1) p)=3$ and the linear series $|(g-1) p|$ is base-point-free (see Corollary 4.5). We shall describe later how this fact is involved in the application to triply periodic minimal surface.

As far as higher values of $r$ are concerned, we prove the sharpness of the bound in Theorem 1.1 for $r=3$ also. Namely,

Theorem 1.3. For any $g \geq 9$, the locus $\mathcal{G}_{g}^{3}$ is non-empty and there exists an irreducible component $\mathcal{Z} \subset \mathcal{G}_{g}^{3}$ of dimension $2 g-4$.

Moreover, we still determine the sequence of integers $h^{0}(C, n p)$ at the general point $[C, p]$ of the irreducible component we construct (see Corollary 4.5). We also study the locus $\mathcal{G}_{g}^{3}$ when $g=9$ and 10, showing that it is reducible and describing an irreducible component exceeding the 'expected dimension' given by Theorem 1.1 (see Section 3).

Theorems 1.2 and 1.3 are analogous to the results in [9, 22] on the sharpness of Harris' bound on the codimension of $\mathcal{M}_{g}^{r}$ in $\mathcal{M}_{g}$. Furthermore, the argument we use to deduce our theorems follows the approach of Farkas, and involves also some of his technical results (see [9, Section 2]). Namely, we argue by induction on $g$ and, beside the description of the base examples, we show that the existence of an irreducible component of $\mathcal{G}_{g-1}^{r}$ having the 'expected dimension' leads to the existence of a component of $\mathcal{G}_{g}^{r}$ still reaching equality in Theorem 1.1] (see Theorem 4.1). In order to prove such a result, we combine Cornalba's theory of spin curves [6] and Eisenbud-Harris' theory of limit linear series [7, 8], and we extend subcanonical points to these settings. In particular, this provides a criterium for smoothing 'limit subcanonical points' and preserving their sequence of Weierstrass gaps (see Corollary 4.4). 
We note finally that the sharpness of the bound on $\mathcal{M}_{g}^{r}$ has been recently proved in [3] for all $r \geq 2$ and $g \geq\left(\begin{array}{c}r+2 \\ 2\end{array}\right)$, according to [9, Conjecture 3.4]. In this line, it seems natural to conjecture the sharpness of the bound in Theorem 1.1 for the very same values of $r$ and $g$.

The original motivation of this work was the study of triply periodic minimal surfaces in the 3dimensional Euclidean space $\mathbb{R}^{3}$. They are very classical geometric objects, which have been studied long since (see e.g. [21, 16]). We say that a compact connected Riemann surface $M$ of genus $g \geq 3$ is periodic if admits a conformal minimal immersion $\iota: M \longrightarrow \mathbb{T}^{3}$ into a flat 3 -dimensional torus $\mathbb{T}^{3}=\mathbb{R}^{3} / \Lambda$. Then a proper triply periodic minimal surface $S$ in $\mathbb{R}^{3}$ is the inverse image under the universal covering $\mathbb{R}^{3} \longrightarrow \mathbb{T}^{3}$ of some immersed minimal surface $\iota(M) \subset \mathbb{T}^{3}$. Following [20], we discuss two related problems on this topic. On one hand, we are aimed at describing the locus $\mathcal{R}_{g}$ of isomorphism classes of periodic surfaces $[M] \in \mathcal{M}_{g}$. On the other hand, we investigate the existence of minimal surfaces in any flat 3-dimensional real torus.

By Generalized Weierstrass Representation (see e.g. [2]), each periodic surface $M$ carries a theta-characteristic - or, equivalently a spin structure $-\mathcal{L}$ such that $h^{0}(M, \mathcal{L}) \geq 2$ and the linear series $|\mathcal{L}|$ is base-point-free. Thus the locus $\mathcal{R}_{g}$ consists of two disjoint loci $\mathcal{R}_{g}^{\text {even }} \subset \mathcal{M}_{g}^{1}$ and $\mathcal{R}_{g}^{\text {odd }} \subset \mathcal{M}_{g}^{2}$ parameterizing even and odd periodic surfaces, respectively. We also denote by $\mathcal{R}_{g}^{\text {hyp }}$ the locus of hyperelliptic periodic surfaces, and we say that a proper triply periodic minimal surface in $\mathbb{R}^{3}$ is even, odd or hyperelliptic if the underlying periodic surface is.

Furthermore, among Riemann surfaces $M$ possessing a theta-characteristic $\mathcal{L}$ as above, periodic surfaces are characterized by a particular condition on their real periods. In [20], the latter condition has been interpreted in terms of first order deformations of the associated real period matrix. Using the results in [17] and [19], the existence of periodic surfaces in $\mathcal{M}_{g}$ follows from the existence of pointed curves $[C, p] \in \mathcal{G}_{g} \subset \mathcal{M}_{g, 1}$ such that $h^{0}(C,(g-1) p) \geq 2$ and the linear series $|(g-1) p|$ is base-point-free. Thus the description of the subcanonical loci $\mathcal{G}_{g}^{1}, \mathcal{G}_{g}^{2}$ and $\mathcal{G}_{g}^{\text {hyp }}$ leads to the following density result, which extends [20, Theorem 1] to odd periodic surfaces of genus $g \geq 7$.

Theorem 1.4. The following hold:

(i) If $g \geq 3$, then $\mathcal{R}_{g}^{\text {even }}$ is dense in $\mathcal{M}_{g}^{1}$.

(ii) If $g \geq 6$, then $\mathcal{R}_{g}^{\text {odd }}$ is dense in $\mathcal{M}_{g}^{2}$.

(iii) If $g \geq 3$ and $g$ is odd, then $\mathcal{R}_{g}^{\text {hyp }}$ is dense in the hyperelliptic locus $\mathcal{M}_{g}^{\text {hyp }}$.

Analogously, by means of the very same argument of [20, Theorem 2] relying on isogenies between flat 3-dimensional tori, we achieve the following existence result.

Theorem 1.5. Any flat 3-dimensional torus contains countably many distinct genus $g$ immersed compact even minimal surfaces if $g \geq 3$, odd minimal surfaces if $g \geq 6$, and hyperelliptic minimal surfaces if $g \geq 3$ is odd.

The 3-dimensional Euclidean space contains countably many 6-dimensional families of genus $g$ proper triply periodic even minimal surfaces if $g \geq 3$, odd minimal surfaces if $g \geq 6$, and hyperelliptic minimal surfaces if $g \geq 3$ is odd.

The paper is organized as follows. In Section 2 we recall some basic facts and we prove preliminary results involved in the analysis of subcanonical loci in $\mathcal{M}_{g, 1}$. Section 3 mainly concerns the description of the loci $\mathcal{G}_{6}^{2}$ and $\mathcal{G}_{9}^{3}$, which are the base cases to develop our inductive argument. Section 4 is devoted to prove Theorems 1.1, 1.2, 1.3 and certain additional results concerning the 
subcanonical locus and its boundary in $\overline{\mathcal{M}}_{g, 1}$. Finally, in Section 5 we turn to periodic minimal surfaces and, after connecting them to theta-characteristics, we retrace the argument of [20] to prove Theorems 1.4 and 1.5 .

\section{PReliminaries}

This section concerns the preliminary notions and results necessary to discuss the loci $\mathcal{G}_{g}^{r}$ and to prove the related theorems we stated in the Introduction. In particular, we shall deal with Weierstrass points, limit linear series and limits of theta-characteristics, with a view toward subcanonical points.

Notation. We work throughout over the field $\mathbb{C}$ of complex numbers, unless otherwise stated. By curve we mean a complete connected reduced algebraic curve over $\mathbb{C}$. We denote by $h^{0}(C, \mathcal{L})$ the dimension of the space $H^{0}(C, \mathcal{L})$ of global sections of a line bundle $\mathcal{L}$ on a curve $C$.

2.1. Weierstrass points. Let $C$ be a smooth curve of genus $g$ and let $L=(\mathcal{L}, V)$ be a $\mathfrak{g}_{d}^{r}$ on $C$, that is a line bundle $\mathcal{L}$ of degree $d$ endowed with a $(r+1)$-dimensional subspace $V \subset H^{0}(C, \mathcal{L})$. As in [7], given a point $p \in C$, the set $\left\{\operatorname{ord}_{p}(s) \mid s \in V\right\}$ of orders of vanishing of sections of $V$ at $p$ consists of exactly $r+1$ distinct integers $0 \leq a_{0}^{L}(p)<a_{1}^{L}(p)<\cdots<a_{r}^{L}(p) \leq d$, and the sequence $a^{L}(p):=\left(a_{0}^{L}(p), a_{1}^{L}(p), \ldots, a_{r}^{L}(p)\right)$ is called the vanishing sequence of $L$ at $p$. The ramification sequence of $L$ at $p$ is the sequence $\alpha^{L}(p):=\left(\alpha_{0}^{L}(p), \alpha_{1}^{L}(p), \ldots, \alpha_{r}^{L}(p)\right)$ such that $\alpha_{i}^{L}(p):=a_{i}^{L}(p)-i$ for any $0 \leq i \leq r$, and the weight of $p$ with respect to $L$ is defined as the integer

$$
w^{L}(p):=\sum_{i=0}^{r} \alpha_{i}^{L}(p)
$$

In the case of the canonical series $K_{C}:=\left(\omega_{C}, H^{0}\left(C, \omega_{C}\right)\right)$, the sequence $\left(k_{0}(p), \ldots, k_{g-1}(p)\right)$ such that $k_{i}(p):=a_{i}^{K_{C}}(p)+1$ coincides with the sequence of Weierstrass gaps of $p$, which is the increasing sequence of integers $0 \leq n \leq 2 g-1$ such that $h^{0}\left(C, \mathcal{O}_{C}((n-1) p)\right)=h^{0}\left(C, \mathcal{O}_{C}(n p)\right)$. Moreover, the set of non-gaps $\mathbb{N} \backslash\left\{k_{0}(p), \ldots, k_{g-1}(p)\right\}$ forms a semigroup with respect to addition. We say that a point $p \in C$ is a Weierstrass point of $C$ if its Weierstrass gaps sequence differs from $(1,2, \ldots, g)$. Hence, it follows from the definition that a point $p \in C$ is a subcanonical point if and only if it is a Weierstrass point having $k_{g-1}(p)=2 g-1$, i.e. $a_{g-1}^{K_{C}}(p)=2 g-2$ and $\alpha_{g-1}^{K_{C}}(p)=g-1$.

Finally, let $\mathcal{M}_{g, 1}$ denote the moduli space of pointed smooth curves of genus $g$, and let $\alpha=\left(\alpha_{0}, \ldots, \alpha_{g-1}\right)$ be the ramification sequence of the canonical series $K_{C}$ at a point $p \in C$, with $[C, p] \in \mathcal{M}_{g, 1}$. Then every component of the locally closed subset $\mathcal{C}_{\alpha}=$ $\left\{[Y, y] \in \mathcal{M}_{g, 1} \mid \alpha_{i}^{K_{Y}}(y)=\alpha_{i}\right.$ for all $\left.0 \leq i \leq g-1\right\}$ has codimension at most $w^{K_{C}}(p)=\sum_{i=0}^{g-1} \alpha_{i}$ (see e.g. [8]).

Remark 2.1. The bound on the codimension of $\mathcal{C}_{\alpha}$ could lead to an estimate of $\operatorname{dim} \mathcal{G}_{g}^{r}$. According to the semigroup condition, the ramification sequence of minimal weight for a point $[C, p] \in \mathcal{G}_{g}^{r}$ is

$$
\alpha=(\underbrace{0, \ldots, 0}_{g-r-1}, \underbrace{r, \ldots, r}_{r}, g-1) .
$$


Then the existence of a pair $(C, p)$ having such a ramification sequence would give $\operatorname{dim} \mathcal{G}_{g}^{r}=\operatorname{dim} \mathcal{C}_{\alpha} \geq$ $3 g-2-w^{K_{C}}(p)=2 g-1-r^{2}$. In [4], it has been proved that the ramification sequences of a general point of $\mathcal{G}_{g}^{0}$ and $\mathcal{G}_{g}^{1}$ does satisfy (2.1). For $r=0$, we then get $\operatorname{dim} \mathcal{G}_{g}^{0} \geq 2 g-1$, which is actually an equality. However, in the light of Theorem 2.1, the bound above turns out to be no longer sharp as $r \geq 1$ (see also [8, p. 497] and [4, Remark 2]).

2.2. Limit linear series. Following [7, we briefly review the notion of limit linear series on curves of compact type. In particular, we focus on nodal curves of the form $X=C_{1} \cup_{q} C_{2}$ given by two smooth curves $C_{1}$ and $C_{2}$ of genus $g-j$ and $j$ respectively, meeting at a single ordinary node $q$.

A crude limit $\mathfrak{g}_{d}^{r}$ on $X$ is a collection $L=\left\{\left(\mathcal{L}_{C_{1}}, V_{C_{1}}\right),\left(\mathcal{L}_{C_{2}}, V_{C_{2}}\right)\right\}$, where $L_{C_{1}}:=\left(\mathcal{L}_{C_{1}}, V_{C_{1}}\right)$ and $L_{C_{2}}:=\left(\mathcal{L}_{C_{2}}, V_{C_{2}}\right)$ are $\mathfrak{g}_{d}^{r}$ on $C_{1}$ and $C_{2}$ respectively, such that the vanishing sequences $a^{L_{C_{1}}}(q)$ and $a^{L_{C_{2}}}(q)$ satisfy the compatibility conditions

$$
a_{i}^{L_{C_{1}}}(q)+a_{r-i}^{L_{C_{2}}}(q) \geq d \quad \text { for any } 0 \leq i \leq r .
$$

In particular, given a point $p \in C_{2}-\{q\}$ (resp. $p \in C_{1}-\{q\}$ ), we define the vanishing and the ramification sequences of $L$ at $p$ as $a^{L}(p):=a^{L_{C_{2}}}(p)$ and $\alpha^{L}(p):=\alpha^{L_{C_{2}}}(p)\left(\right.$ resp. $a^{L}(p):=a^{L_{C_{1}}}(p)$ and $\left.\alpha^{L}(p):=\alpha^{L_{C_{1}}}(p)\right)$. Moreover, we say that $L$ is a refined limit $\mathfrak{g}_{d}^{r}$, or simply a limit $\mathfrak{g}_{d}^{r}$, if all the inequalities in (2.2) are equalities.

It is worth recalling that in [7, Section 3] are constructed the scheme of limit $\mathfrak{g}_{d}^{r}$ on a curve of compact type $X$, and more generally schemes parameterizing limit $\mathfrak{g}_{d}^{r}$ varying on families of pointed curves of compact type.

Remark 2.2. Let $(X, p)$ be a pointed singular curve of compact type and let $L$ be a limit $\mathfrak{g}_{d}^{r}$ as above. Suppose that the triple $(X, p, L)$ is smoothable, that is there exists a family of smooth pointed curves endowed with a $\mathfrak{g}_{d}^{r}$ having $(X, p, L)$ as central fibre. Denoting by $B$ the base space of the family and by $\left(X_{b}, p_{b}, L_{b}\right)$ the fibre over $b \in B$, we have that the vanishing and the ramification sequences of $L_{b}$ at $p_{b} \in X_{b}$ satisfy upper semi-continuity, that is $a_{i}^{L_{b}}\left(p_{b}\right) \leq a_{i}^{L}(p)$ and $\alpha_{i}^{L_{b}}\left(p_{b}\right) \leq \alpha_{i}^{L}(p)$ for any $0 \leq i \leq r$ and for any $b \in B$.

We recall that $\mathcal{M}_{g}^{r}$ is the sublocus of $\mathcal{M}_{g}$ which parameterizes curves $C$ admitting a thetacharacteristic $L$ such that $h^{0}(C, L) \geq r+1$ and $h^{0}(C, L) \equiv r+1(\bmod 2)$. The following lemma describes 'limit theta-characteristics' in terms of limit $\mathfrak{g}_{g-1}^{r}$ on curves lying in the boundary of $\overline{\mathcal{M}}_{g}^{r}$ (see [9, Lemma 2.2]).

Lemma 2.3. Let $\left[C_{1} \cup_{q} C_{2}\right] \in \overline{\mathcal{M}}_{g}^{r}$. Then the curve $C_{1} \cup_{q} C_{2}$ possesses a limit $\mathfrak{g}_{g-1}^{r}$ $L=\left\{\left(\mathcal{L}_{C_{1}}, V_{C_{1}}\right),\left(\mathcal{L}_{C_{2}}, V_{C_{2}}\right)\right\}$ such that

$$
\mathcal{L}_{C_{1}}^{2} \cong \omega_{C_{1}}(2 j q) \quad \text { and } \quad \mathcal{L}_{C_{2}}^{2} \cong \omega_{C_{2}}(2(g-j) q),
$$

where $g-j$ and $j$ are the genus of $C_{1}$ and $C_{2}$, respectively.

Remark 2.4. Given a smooth pointed curve $[Y, y] \in \mathcal{M}_{g, 1}$, the point $y \in Y$ is subcanonical if and only if there exists some complete $\mathfrak{g}_{g-1}^{r} L$ on $Y$ such that $L^{2} \cong \omega_{Y}$ and $a_{r}^{L}(y)=g-1$. In the previous lemma, conditions (2.3) extend the notion of theta-characteristic to limit $\mathfrak{g}_{g-1}^{r}$ on nodal curves (see also [7, p. 363]). Analogously, upper semi-continuity gives that for any $\left[C_{1} \cup_{q} C_{2}, p\right] \in \overline{\mathcal{G}}_{g}^{r}$, the curve $C_{1} \cup_{q} C_{2}$ possesses a limit $\mathfrak{g}_{g-1}^{r} L$ satisfying (2.3) and the extra condition $a_{r}^{L}(p)=g-1$. 
Let $\Delta_{1,1} \subset \overline{\mathcal{M}}_{g, 1}$ denote the irreducible boundary component whose general point $\left[C \cup_{q} E, p\right]$ is such that $C$ has genus $g-1, E$ is an elliptic curve and $p \in E$. In the spirit of [4, Lemma 2.2], we prove the following lemma, which describes pointed curves on $\Delta_{1,1}$ having a 'limit subcanonical point' whose associated 'limit theta-characteristic' has $r+1$ sections.

Lemma 2.5. Fix $g \geq 3$ and $r \geq 1$, and let $\left[C \cup_{q} E, p\right] \in \Delta_{1,1} \subset \overline{\mathcal{M}}_{g, 1}$.

(i) Let $L=\left\{\left(\mathcal{L}_{C}, V_{C}\right),\left(\mathcal{L}_{E}, V_{E}\right)\right\}$ be a limit $\mathfrak{g}_{g-1}^{r}$ on $C \cup_{q} E$ satisfying

$$
\mathcal{L}_{C}^{2} \cong \omega_{C}(2 q) \quad \text { and } \quad \mathcal{L}_{E}^{2} \cong \omega_{E}(2(g-1) q),
$$

and such that $a_{r}^{L}(p)=g-1$. Then $q \in C$ is a subcanonical point with $h^{0}(C,(g-2) q) \geq r$, and $p-q \in E$ is a torsion point of order $2 g-2$.

(ii) Conversely, if $q \in C$ is a subcanonical point with $h^{0}(C,(g-2) q) \geq r+1$, and $p-q \in$ $E$ is a torsion point of order exactly $2 g-2$, then the curve $C \cup_{q} E$ admits a limit $\mathfrak{g}_{g-1}^{r}$ $L=\left\{\left(\mathcal{L}_{C}, V_{C}\right),\left(\mathcal{L}_{E}, V_{E}\right)\right\}$ satisfying conditions (2.4) and such that $a_{r}^{L}(p)=g-1$.

In particular, $\mathcal{L}_{C}=\mathcal{O}_{C}((g-1) q)$ and $\mathcal{L}_{E}=\mathcal{O}_{E}((g-1) p)$ in both (i) and (ii).

Proof. In order to prove (i), we note that $L_{E}=\left(\mathcal{L}_{E}, V_{E}\right)$ is a $\mathfrak{g}_{g-1}^{r}$ on $E$ having vanishing order $a_{r}^{L_{E}}(p)=a_{r}^{L}(p)=g-1$ at $p \in E$. Thus there exists $\sigma_{E} \in V_{E}$ such that $\operatorname{ord}_{p}\left(\sigma_{E}\right)=g-1$, and hence $\mathcal{O}_{E}((g-1) p) \cong \mathcal{L}_{E}$. Thanks to conditions (2.4) we have $\mathcal{O}_{E}((2 g-2) p) \cong \mathcal{L}_{E}^{2} \cong \mathcal{O}_{E}((2 g-2) q)$, that is $p-q \in E$ is a torsion point of order $2 g-2$.

Since $\operatorname{ord}_{p}\left(\sigma_{E}\right)=g-1$, we have $\operatorname{ord}_{q}\left(\sigma_{E}\right)=0$ and hence $a_{0}^{L_{E}}(q)=0$. Then $a_{r}^{L_{C}}(q)=g-1$ by compatibility conditions (2.2). In particular, $\mathcal{O}_{C}((g-1) q) \cong \mathcal{L}_{C}$ and $h^{0}(C,(g-1) q) \geq \operatorname{dim} V_{C}=$ $r+1$. Therefore $h^{0}(C,(g-2) q) \geq r$ and $\mathcal{O}_{C}((2 g-2) q) \cong \mathcal{L}_{C}^{2} \cong \omega_{C}(2 q)$ by (2.4). Thus $(2 g-4) q$ is a canonical divisor of the curve $C$ of genus $g-1$, i.e. $q \in C$ is a subcanonical point and assertion (i) follows.

So we turn to prove (ii). Let $\mathcal{L}_{C}:=\mathcal{O}_{C}((g-1) q)$ and $\mathcal{L}_{E}:=\mathcal{O}_{E}((g-1) p)$. By assumption, $(2 g-2) p$ and $(2 g-2) q$ are linearly equivalent divisors on $E$, and $(2 g-4) q$ is a canonical divisor on $C$. Thus $\mathcal{L}_{C}$ and $\mathcal{L}_{E}$ satisfy conditions (2.4). Moreover, the subcanonical point $q \in C$ is such that $h^{0}(C,(g-2) q) \geq r+1$. Then there exists $\sigma \in H^{0}\left(C, \mathcal{O}_{C}((g-2) q)\right)$ satisfying $\operatorname{ord}_{q}(\sigma)=g-2$, and we can choose a $(r+1)$-dimensional subspace $V \subset H^{0}\left(C, \mathcal{O}_{C}((g-2) q)\right)$ passing through $\sigma$. We define $V_{C} \subset H^{0}\left(C, \mathcal{O}_{C}((g-1) q)\right)$ as the image of $V$ under the natural map $H^{0}\left(C, \mathcal{O}_{C}((g-2) q)\right) \hookrightarrow H^{0}\left(C, \mathcal{O}_{C}((g-1) q)\right)$. So the linear series $L_{C}:=\left(\mathcal{L}_{C}, V_{C}\right)$ has a base point at $q$, and its vanishing sequence $a^{L_{C}}(q)=\left(c_{0}, \ldots, c_{r}\right)$ at $q$ is such that $c_{0} \geq 1$ and $c_{r}=g-1$.

We consider the sequences $\left(b_{0}, \ldots, b_{r}\right)$ and $\left(a_{0}, \ldots, a_{r}\right)$ defined as $b_{i}:=g-1-c_{r-i}$ for any $0 \leq i \leq r$, $a_{r}:=g-1$ and $a_{r-i}:=g-2-b_{i}$ for any $1 \leq i \leq r$. Then [8, Proposition 5.2] assures that if

$$
\begin{array}{lll}
a_{r-i}+b_{i}=g-1 & \Rightarrow & a_{r-i} p+b_{i} q \sim(g-1) p \\
a_{r-i} p+\left(b_{i}+1\right) q \sim(g-1) p & \Rightarrow & b_{i}+1=b_{i+1}
\end{array}
$$

hold for each $i$, there exists a linear series $L_{E}:=\left(\mathcal{L}_{E}, V_{E}\right)$ such that $a^{L_{E}}(p)=\left(a_{0}, \ldots, a_{r}\right)$ and $a^{L_{E}}(q)=\left(b_{0}, \ldots, b_{r}\right)$. Since $p-q$ is a torsion point on $E$ having order exactly $2 g-2$, conditions (2.5) are satisfied. In particular, $a_{r}^{L}(p)=a_{r}=g-1$. Moreover, $b_{i}+c_{r-i}=g-1$ for any $0 \leq i \leq r$ by definition, therefore $L$ is a refined limit linear series. 
2.3. Theta-characteristics and spin curves. We summarize the approach of [6] to compactify the moduli space $\mathcal{S}_{g}$ parameterizing isomorphism classes $[C, \mathcal{L}]$, where $[C] \in \mathcal{M}_{g}$ and $\mathcal{L}$ is a thetacharacteristic on $C$. To this aim, we consider a semistable curve $X$ having at most ordinary nodes as singularities and arithmetic genus $p_{a}(X)=g \geq 2$. An exceptional component is a smooth rational curve $R \subset X$ meeting the rest of the curve $X$ at two points. We say that $X$ is a decent curve if every two exceptional components are disjoint. A spin curve is a triple $(X, \xi, \alpha)$ consisting of a decent curve $X$, a line bundle $\xi$ of degree $g-1$ on $X$ such that $\xi_{\mid R}=\mathcal{O}_{R}(1)$ for any exceptional component $R \subset X$, and a homomorphism $\alpha: \xi^{2} \longrightarrow \omega_{X}$ being non-null on every non-exceptional component of $X$. In particular, when $X$ is a curve of compact type, the restriction $\xi_{\mid C}$ gives a thetacharacteristic on any smooth non-exceptional component $C \subset X$. Moreover, any decent curve is obtained by blowing-up some nodes of a stable curve $[\widetilde{X}] \in \overline{\mathcal{M}}_{g}$. Thus we define the moduli space $\overline{\mathcal{S}}_{g}$ parameterizing isomorphism classes $[X, \xi, \alpha]$ of spin curves, and there exists a finite map

$$
\pi: \overline{\mathcal{S}}_{g} \longrightarrow \overline{\mathcal{M}}_{g}
$$

sending $[X, \xi, \alpha]$ to the class of the stable model $[\widetilde{X}]$.

Remark 2.6. Consider a stable curve $\tilde{X}=C_{1} \cup_{q} C_{2}$ whose components are two smooth curves of genus $g-j$ and $j$ meeting at a node $q$. Then $\widetilde{X}$ does not admit any structure of spin curve, and the fibre $\pi^{-1}[\widetilde{X}]$ consists of classes $[X, \xi, \alpha] \in \overline{\mathcal{S}}_{g}$, where $X=C_{1} \cup_{q^{\prime}} R \cup_{q^{\prime \prime}} C_{2}$ is the blow-up of $\tilde{X}$ at $q$ (see [6, Example 3.1]).

A family of spin curves is a triple $(\mathcal{X} \stackrel{\phi}{\longrightarrow} T, \xi, \alpha)$ consisting of a family of decent curves $\mathcal{X} \longrightarrow T$, a line bundle $\xi$ on $\mathcal{X}$, and a homomorphism $\alpha: \xi^{2} \longrightarrow \omega_{\phi}$ such that each triple $\left(X_{t}, \xi_{t}, \alpha_{t}\right)$ is a spin curve, where $X_{t}:=\phi^{-1}(t), \xi_{t}:=\xi_{\mid X_{t}}$ and $\alpha_{t}:=\alpha_{\mid \xi_{t}^{2}}$. The following theorem extends to spin curves two classical results on theta-characteristics due to Mumford and Harris, respectively (see [12, 18] and [5, Theorem 3.3]).

Theorem 2.7. Let $(\mathcal{X} \stackrel{\phi}{\longrightarrow} T, \xi, \alpha)$ be a family of spin curves. Then

(i) the function $T \longrightarrow \mathbb{Z} / 2 \mathbb{Z}$ defined as $t \longmapsto\left[h^{0}\left(C_{t}, \xi_{t}\right)\right]_{(\bmod 2)}$ is locally constant;

(ii) the locus $T^{r}:=\left\{t \in T \mid h^{0}\left(X_{t}, \xi_{t}\right) \geq r+1\right.$ and $\left.h^{0}\left(X_{t}, \xi_{t}\right) \equiv r+1(\bmod 2)\right\}$ is either empty or it has codimension at most $\left(\begin{array}{c}r+1 \\ 2\end{array}\right)$.

In particular, the locus

$$
\mathcal{S}_{g}^{r}:=\left\{[C, \mathcal{L}] \in \mathcal{S}_{g} \mid h^{0}(C, \mathcal{L}) \geq r+1 \text { and } h^{0}(C, \mathcal{L}) \equiv r+1(\bmod 2)\right\}
$$

has codimension at most $\left(\begin{array}{c}r+1 \\ 2\end{array}\right)$ in $\mathcal{S}_{g}$, according to the identity $\mathcal{M}_{g}^{r}=\pi\left(\mathcal{S}_{g}^{r}\right)$.

Since we shall mainly deal with pointed curves, let $\mathcal{S}_{g, 1}$ denote the moduli space of pointed smooth spin curves, and let $\mathcal{S}_{g, 1}^{r}$ be the pre-image of $\mathcal{S}_{g}^{r}$ under the forgetful morphism $\mathcal{S}_{g, 1} \longrightarrow \mathcal{S}_{g}$. We recall that a theta-characteristic $\mathcal{L}$ on a curve $C$ is said to be odd (resp. even) if $h^{0}(C, \mathcal{L}$ ) is. Moreover, the locus $\Delta_{1,1} \subset \overline{\mathcal{M}}_{g, 1}$ denotes the boundary divisor parameterizing nodal curves having an elliptic tail with a marked point. Then we define the irreducible boundary components $A_{1,1}^{+}$and $A_{1,1}^{-}$of $\overline{\mathcal{S}}_{g}$ as

$$
\left.A_{1,1}^{+}:=\begin{array}{l|l}
{[X, \xi, \alpha, p] \in \overline{\mathcal{S}}_{g}} & \begin{array}{l}
\pi([X, \xi, \alpha, p])=\left[C \cup_{q} E, p\right] \in \Delta_{1,1}, \\
\xi_{\mid C} \text { and } \xi_{\mid E} \text { are even }
\end{array}
\end{array}\right\}
$$


and

$$
A_{1,1}^{-}:=\left\{\begin{array}{l|l}
{[X, \xi, \alpha, p] \in \overline{\mathcal{S}}_{g}} & \begin{array}{l}
\pi([X, \xi, \alpha, p])=\left[C \cup_{q} E, p\right] \in \Delta_{1,1}, \\
\xi_{\mid C} \text { is odd and } \xi_{\mid E} \text { is even }
\end{array}
\end{array}\right\} .
$$

Hence the partial compactification $\mathcal{S}_{g}^{*}:=\mathcal{S}_{g} \cup A_{1,1}^{+} \cup A_{1,1}^{-}$maps finitely over $\mathcal{M}_{g} \cup \Delta_{1,1}$ through $\pi$. Finally, we have the following result (see [9, Proposition 2.4]).

Lemma 2.8. Let $\left[C \cup_{q} E, p\right] \in \Delta_{1,1}$ such that $[C, q] \in \mathcal{G}_{g-1}^{r}$ and $p-q \in E$ is a torsion point of order exactly $2 g-2$. Let $[X, \xi, \alpha] \in \overline{\mathcal{S}}_{g}$ be the spin curve such that $X=C \cup_{q^{\prime}} R \cup_{q^{\prime \prime}} E$ is the blow up of $C \cup_{q} E$ at $q, \xi_{\mid C}=\mathcal{O}_{C}\left((2 g-4) q^{\prime}\right)$ and $\xi_{\mid E}=\mathcal{O}_{E}\left((g-1)\left(p-q^{\prime \prime}\right)\right)$. If $\left[C, \xi_{\mid C}\right] \in \mathcal{S}_{g-1}$ lies on an irreducible component of $\mathcal{S}_{g-1}^{r}$ having codimension $\left(\begin{array}{c}r+1 \\ 2\end{array}\right)$ in $\mathcal{S}_{g-1}$, then $[X, \xi, \alpha] \in \overline{\mathcal{S}}_{g}^{r}$ and $\left[C \cup_{q} E\right] \in \overline{\mathcal{M}}_{g}^{r}$.

Remark 2.9. Lemma 2.8 is actually included in the proof of [9, Proposition 2.4], under slightly different assumptions. In particular, the point $q$ is assumed to be general on both $C$ and $E$, and $\xi_{\mid E}=\mathcal{O}_{E}(t-s)$ for some torsion point of order two $t-s \in E$. However, these differences do not affect the proof of Lemma 2.8.

\section{ExAmples}

This section is mainly devoted to describe the loci $\mathcal{G}_{6}^{2}$ and $\mathcal{G}_{9}^{3}$. On one hand, they provide the first step of the inductive argument we shall perform in the next section. On the other hand, we shall see that $\mathcal{G}_{9}^{3}$ is reducible, and we shall describe a component satisfying strict inequality in Theorem 1.1. Moreover, we shall briefly discuss the locus $\mathcal{G}_{10}^{3}$, which possesses two distinct irreducible component having the 'expected dimension'.

Example $3.1(r=2$ and $g=6)$. It follows from the detailed analysis in [4, Section 4.5] that $\mathcal{G}_{6}^{2} \subset \mathcal{M}_{6,1}$ is an irreducible non-empty locus of dimension 10 , and for any point $[C, p] \in \mathcal{G}_{6}^{2}$, the canonical ramification sequence at $p$ is $\alpha^{K_{C}}(p)=(0,0,0,2,2,5)$. In particular, both the dimension of $\mathcal{G}_{6}^{2}$ and the weight of $\alpha^{K_{C}}(p)$ reach the smallest possible values in view of Theorem 1.1 and Remark 2.1.

We note further that each point $[C, p] \in \mathcal{G}_{6}^{2}$ is given by a smooth curve $C \in \mathbb{P}^{2}$ of degree $d=5$ having a 5 -fold inflection point at $p \in C$, that is there exists a line $\ell \in \mathbb{P}^{2}$ such that $(C \cdot \ell)_{p}=5$. Conversely, given such a quintic plane curve $C$, we have that $\omega_{C} \cong \mathcal{O}_{C}(2)$ and $\left|\mathcal{O}_{C}(1)\right|$ is the unique $\mathfrak{g}_{5}^{2}$ on $C$, so that any 5-fold inflection point $p \in C$ is subcanonical with $h^{0}(C, 5 p)=3$.

In the following examples we turn to describe $\mathcal{G}_{9}^{3}$. We construct two distinct components $\mathcal{Z}_{1}, \mathcal{Z}_{2} \subset \mathcal{G}_{9}^{3}$ of dimension $\operatorname{dim} \mathcal{Z}_{1}=14$ and $\operatorname{dim} \mathcal{Z}_{2}=15$, whose general points $[C, p]$ satisfy $\alpha^{K_{C}}(p)=(0, \ldots, 0,3,3,3,8)$ and $\alpha^{K_{C}}(p)=(0,0,0,1,1,3,3,5,8)$, respectively. In particular, the component $\mathcal{Z}_{1}$ satisfies equality in Theorem 1.1, and the canonical sequence at its general point has minimal weight. On the other hand, the component $\mathcal{Z}_{2}$ exceeds the 'expected dimension'.

Example $3.2(r=3$ and $g=9)$. Let $\mathcal{Z}_{1} \subset \mathcal{M}_{9,1}$ be the locus whose general point $[C, p]$ is such that $C=S \cap T \subset \mathbb{P}^{3}$ is the complete intersection of a smooth quadric $S$ and a smooth quartic $T$, where $p \in C$ is an inflection point of order 8, i.e. there exists a plane $H \subset \mathbb{P}^{3}$ satisfying $(C \cdot H)_{p}=8$.

By adjunction formula, any such a curve $C$ has genus 9 and $\omega_{C} \cong \mathcal{O}_{C}(2)$, so that $p \in C$ is a subcanonical point. It follows from the description of [1, p. 199-200] that the sequence of Weierstrass gaps of $p$ is either $(1, \ldots, 5,9,10,11,17)$ or $(1,2,3,5,6,9,10,13,17)$. If it were the latter, then $|4 p|$ 
would be the $\mathfrak{g}_{4}^{1}$ obtained by projecting $C$ from its tangent line $\ell$ at $p$. Therefore $H \cap S=2 \ell$ and $S$ would be a quadric cone, contradicting the assumption of smoothness. Thus the sequence of Weierstrass gaps at $p$ is the former and the corresponding canonical ramification sequence is $\alpha^{K_{C}}(p)=(0, \ldots, 0,3,3,3,8)$. In particular, $h^{0}(C, 8 p)=4$ and hence $[C, p] \in \mathcal{G}_{9}^{3}$.

The existence of smooth curves as above can be proved as follows. We fix in $\mathbb{P}^{3}$ a smooth quadric $S$ and a plane $H$ such that $D:=S \cap H$ is a smooth curve. Then $S \cong \mathbb{P}^{1} \times \mathbb{P}^{1}$ and $D$ has bidegree $(1,1)$ on $S$. In particular, $D$ is a smooth plane conic, so that $D \cong \mathbb{P}^{1}$. Thus $\mathcal{O}_{S}(4) \cong \mathcal{O}_{\mathbb{P}^{1} \times \mathbb{P}^{1}}(4,4)$ and $\mathcal{O}_{D}(4) \cong \mathcal{O}_{\mathbb{P}^{1}}(8)$. Moreover, the restriction maps $H^{0}\left(\mathbb{P}^{3}, \mathcal{O}_{\mathbb{P}^{3}}(4)\right) \longrightarrow H^{0}\left(S, \mathcal{O}_{S}(4)\right)$ and $H^{0}\left(\mathbb{P}^{1} \times \mathbb{P}^{1}, \mathcal{O}_{\mathbb{P}^{1} \times \mathbb{P}^{1}}(4,4)\right) \longrightarrow H^{0}\left(\mathbb{P}^{1}, \mathcal{O}_{\mathbb{P}^{1}}(8)\right)$ turn out to be surjective. Hence all the effective divisors of degree 8 on $D$ are cut out by curves of bidegree $(4,4)$ on $S$. Therefore, fixing a point $p \in D$, there exists some curve $C \subset S$ of bidegree $(4,4)$ meeting $D$ at $p$ with multiplicity 8 , so that $(C \cdot H)_{p}=8$ as well. Furthermore, the surjections above show that $C$ must be the complete intersection of $S$ with some quartic surface $T \subset \mathbb{P}^{3}$. Finally, let $C \subset \mathbb{P}^{3}$ be the complete intersection of $S:=\left\{x z-y^{2}+t^{2}=0\right\}$ and $T:=\left\{x^{4}+x^{3} z-x^{2} y^{2}+x^{2} y z-x y^{3}+x^{2} z^{2}+x y^{2} z-2 y^{4}+x y z^{2}-\right.$ $\left.y^{3} z+x z^{3}-y^{2} z^{2}+t z^{3}+t^{4}=0\right\}$. By direct computation, it is possible to check that $C, S$ and $T$ are smooth. Moreover, the plane $H:=\{t=0\}$ and the point $p:=(0: 0: 1: 0)$ are such that $(C \cdot H)_{p}=8$. Hence the linear subsystem $|V| \subset\left|\mathcal{O}_{S}(4)\right|$ of curves $C \subset S$ satisfying the linear condition $(C \cdot H)_{p} \geq 8$ is non-empty, and its general element is smooth by Bertini's Theorem. In particular, the locus $\mathcal{Z}_{1}$ is non-empty as well.

To conclude, we point out that $(C \cdot H)_{p} \geq 8$ imposes exactly 8 independent linear conditions on $\left|\mathcal{O}_{S}(4)\right|$. Thus the dimension of $\mathcal{Z}_{1}$ is

$$
\begin{aligned}
\operatorname{dim} \mathcal{Z}_{1}= & \underbrace{\left(\begin{array}{c}
2+3 \\
3
\end{array}\right)-1}_{\text {choice of } S}+\underbrace{\left(\begin{array}{c}
4+3 \\
3
\end{array}\right)-\left(\begin{array}{c}
2+3 \\
3
\end{array}\right)-1}_{\text {choice of } T_{\mid S}}+\underbrace{1}_{\text {choice of } p}+\underbrace{3}_{\text {choice of } H} \\
& -\underbrace{8}_{(C \cdot H)_{p} \geq 8}-\underbrace{15}_{\text {PGL }(4)}=14 .
\end{aligned}
$$

Example $3.3(r=3$ and $g=9)$. We define $\mathcal{Z}_{2} \subset \mathcal{M}_{9,1}$ as the locus of pointed curves $[C, p]$ such that $C=S \cap T \subset \mathbb{P}^{3}$ is the complete intersection of a quadric cone $S$ and a smooth quartic $T$, and there exists a line of the ruling $\ell \subset S$ satisfying $(C \cdot \ell)_{p}=4$.

By arguing as above, it is possible to check that $\mathcal{Z}_{2}$ is a non-empty component of $\mathcal{G}_{9}^{3}$ having $\operatorname{dim} \mathcal{Z}_{2}=15$ and $\alpha^{K_{C}}(p)=(0,0,0,1,1,3,3,5,8)$ at its general point. Furthermore, the component $\mathcal{Z}_{1}$ is not contained in $\mathcal{Z}_{2}$ because of upper semi-continuity of ramification sequences.

To conclude, we briefly describe also the locus $\mathcal{G}_{10}^{3}$, which is reducible and contains two irreducible components satisfying equality in Theorem 1.1.

Example 3.4 $(r=3$ and $g=10)$. Let $C=\Gamma_{1} \cap \Gamma_{2} \subset \mathbb{P}^{3}$ be a smooth curve obtained as complete intersection of two smooth cubic surfaces $\Gamma_{1}$ and $\Gamma_{2}$. Assume that $p \in C$ is an inflection point of order 9 , and let $H \subset \mathbb{P}^{3}$ be the plane such that $(C \cdot H)_{p}=9$. By adjunction formula, the curve $C$ has genus 10 and $\omega_{C} \cong \mathcal{O}_{C}(2)$, so that $p \in C$ is a subcanonical point and $[C, p] \in \mathcal{G}_{10}^{3}$. Then the configurations of the cubic plane sections $C_{1}:=\Gamma_{1} \cap H$ and $C_{2}:=\Gamma_{2} \cap H$ turn out to distinguish two non-empty components $\mathcal{Z}_{1}, \mathcal{Z}_{2} \subset \mathcal{G}_{10}^{3}$ of dimension $\operatorname{dim} \mathcal{Z}_{1}=\operatorname{dim} \mathcal{Z}_{2}=2 g-4=16$, as follows:

(i) the general point $[C, p] \in \mathcal{Z}_{1}$ is such that the plane cubic $C_{1}$ possesses a 3 -inflection point at $p$, and the canonical ramification sequence is $\alpha^{K_{C}}(p)=(0, \ldots, 0,1,3,3,4,9)$; 
(ii) the general point $[C, p] \in \mathcal{Z}_{2}$ is such that the point $p-O$ on the elliptic curve $C_{1}$ is a torsion point of order 9 for some 3 -inflection point $O \in C_{1}$, and the canonical ramification sequence is $\alpha^{K_{C}}(p)=(0, \ldots, 0,3,3,3,9)$.

Remark 3.5. We point out that, after a detailed analysis, it should be possible to argue as in Example 3.2 and to construct components of $\mathcal{G}_{13}^{4}$ and $\mathcal{G}_{17}^{5}$ having the 'expected dimensions' $2 g-7$ and $2 g-11$, respectively. As it shall be clear in the next section, these constructions would work as base cases of our inductive argument, so that Theorem 1.3 would be extended to both $\mathcal{G}_{g}^{4}$ and $\mathcal{G}_{g}^{5}$. When $r=4$, the component $\mathcal{Z} \subset \mathcal{G}_{13}^{4}$ is described by complete intersections $C=S_{1} \cap S_{2} \cap T \subset \mathbb{P}^{4}$ of two smooth quadric hypersurfaces $S_{1}, S_{2}$ and a cubic hypersurface $T$. When $r=5$, the component $\mathcal{Z} \subset \mathcal{G}_{13}^{4}$ consists instead of complete intersections $C=S_{1} \cap \cdots \cap S_{4} \subset \mathbb{P}^{5}$ of four smooth quadrics.

\section{LOCI OF SUBCANONICAL POINTS}

This section concerns our main results on the subcanonical loci $\mathcal{G}_{g}^{r}$. In particular, after proving Theorem 1.1, we shall present a smoothing theorem, which is the core of the inductive argument to achieve Theorems 1.2 and 1.3. Finally, we shall deduce some further related results.

Proof of Theorem 1.1. Suppose that $\mathcal{G}_{g}^{r}$ is non-empty and let $[C, p] \in \mathcal{G}_{g}^{r}$, so that $p \in C$ is a subcanonical point and $N:=\mathcal{O}_{C}((g-1) p)$ is a theta-characteristic. We want to prove that any irreducible component $\mathcal{Z} \subset \mathcal{G}_{g}^{r}$ passing through $[C, p]$ has $\operatorname{dimension} \operatorname{dim} \mathcal{Z} \geq 2 g-\frac{r(r-1)}{2}-1$. To this aim, let $(\mathcal{C} \stackrel{\phi}{\longrightarrow} U, \mathcal{N}, U \stackrel{\rho}{\longrightarrow} \mathcal{C})$ be a versal deformation of the pointed smooth curve $(C, N, p)$ in $\mathcal{S}_{g, 1}$. Therefore $U / \operatorname{Aut}(C, N, p) \hookrightarrow \mathcal{S}_{g, 1}$, the family $\phi: \mathcal{C} \longrightarrow U$ consists of smooth curves $C_{t}:=\phi^{-1}(t)$ of genus $g$, the line bundle $\mathcal{N}$ on $\mathcal{C}$ is such that $N_{t}:=\mathcal{N}_{\mid C_{t}}$ is a theta characteristic on $C_{t}$, the map $\rho: U \longrightarrow \mathcal{C}$ is a section of $\phi$ with $p_{t}:=\rho(t) \in C_{t}$, and $\left(C_{0}, N_{0}, p_{0}\right)=(C, N, p)$ for some point $0 \in U$.

Then we consider the $(g-1)$-fold relative symmetric product $\mathcal{C}^{(g-1)} \longrightarrow U$ of the family $\mathcal{C}$, so that the fibre over each $t$ is the $(g-1)$-fold symmetric product $C_{t}^{(g-1)}$ of the curve $C_{t}$. Setting $U^{r}:=\left\{t \in U \mid\left[C_{t}, N_{t}, p_{t}\right] \in \mathcal{S}_{g, 1}^{r}\right\}$, we define two subvarieties of $\mathcal{C}^{(g-1)}$ as follows. The first one is

$$
\mathcal{Y}:=\left\{p_{1}+\cdots+p_{g-1} \in C_{t}^{(g-1)} \mid t \in U^{r} \text { and } \mathcal{O}_{C_{t}}\left(p_{1}+\cdots+p_{g-1}\right) \cong N_{t}\right\}
$$

which consists of effective divisors $D_{t} \in C_{t}^{(g-1)}$ that induce the theta-characteristic $N_{t}$ on $C_{t}$ having at least $r+1$ global sections and the same parity as $r+1$. The second subvariety is $\mathcal{P}:=\left\{p_{t}+\cdots+p_{t} \in C_{t}^{(g-1)} \mid t \in U\right\}$, which is the image of the map $\rho^{(g-1)}: U \longrightarrow \mathcal{C}^{(g-1)}$ induced by $\rho$. Therefore, for any $t \in U$ such that $p_{t}+\cdots+p_{t} \in \mathcal{P} \cap \mathcal{Y}$, the line bundle $\mathcal{O}_{C_{t}}\left((g-1) p_{t}\right)$ is a theta-characteristic having $h^{0}\left(C,(g-1) p_{t}\right) \geq r+1$ and $h^{0}\left(C,(g-1) p_{t}\right) \equiv r+1(\bmod 2)$. Thus $\left[C_{t}, p_{t}\right] \in \mathcal{G}_{g}^{r}$.

We note further that the map $U \longrightarrow \mathcal{M}_{g, 1}$ given by $t \longmapsto\left[C_{t}, p_{t}\right]$ is finite. Indeed it factors through the injection $U / \operatorname{Aut}(C, L, p) \hookrightarrow \mathcal{S}_{g, 1}$ and the natural map $\mathcal{S}_{g, 1} \longrightarrow \mathcal{M}_{g, 1}$ defined by $\left[C_{t}, L_{t}, p_{t}\right] \longmapsto\left[C_{t}, p_{t}\right]$. Hence each irreducible component $\mathcal{Z} \subset \mathcal{G}_{g}^{r}$ passing through $[C, p]$ satisfies

$$
\operatorname{dim} \mathcal{Z} \geq \operatorname{dim}(\mathcal{P} \cap \mathcal{Y}) \geq \operatorname{dim} \mathcal{P}+\operatorname{dim} \mathcal{Y}-\operatorname{dim} \mathcal{C}^{(g-1)}
$$

Finally, $\operatorname{since} \operatorname{dim} \mathcal{P}=\operatorname{dim} U=3 g-2, \operatorname{dim} \mathcal{Y} \geq \operatorname{dim} U^{r}+r \geq 3 g-2-\frac{r(r+1)}{2}+r$ by Theorem 2.7, and $\operatorname{dim} \mathcal{C}^{(g-1)}=\operatorname{dim} U+g-1=4 g-3$, we deduce $\operatorname{dim} \mathcal{Z} \geq 2 g-\frac{r(r-1)}{2}-1$ as claimed. 
In analogy with [9, Proposition 2.4], the following result shows that if $\mathcal{G}_{g-1}^{r}$ possesses an irreducible component of the 'expected dimension', its points can be suitably glued to elliptic curves, so that the resulting nodal curves in $\overline{\mathcal{M}}_{g, 1}$ can be smoothed preserving a subcanonical point and the number of sections of the associated theta-characteristic.

Theorem 4.1. Fix $r \geq 1$ and $g \geq 4$. If the locus $\mathcal{G}_{g-1}^{r}$ has an irreducible component $\mathcal{Z}_{g-1}$ of dimension $2 g-\frac{r(r-1)}{2}-3$, then $\mathcal{G}_{g}^{r}$ possesses a non-empty irreducible component $\mathcal{Z}_{g}$ of dimension $2 g-\frac{r(r-1)}{2}-1$.

Proof. We consider the locus

$$
\mathcal{W}:=\left\{\begin{array}{l|l}
{\left[C \cup_{q} E, p\right] \in \overline{\mathcal{M}}_{g, 1}} & \begin{array}{l}
{[C, q] \in \mathcal{Z}_{g-1}, E \text { is an elliptic curve }} \\
p \in E, p-q \in E \text { is a torsion point of } \\
\text { order exactly } 2 g-2
\end{array}
\end{array}\right\} .
$$

Our aim is to prove that $\overline{\mathcal{W}}$ is an irreducible component of the boundary of $\overline{\mathcal{G}}_{g}^{r} \subset \overline{\mathcal{M}}_{g, 1}$. Then we shall deduce the existence of an irreducible component $\mathcal{Z}_{g} \subset \mathcal{G}_{g}^{r}$ whose closure contains $\mathcal{W}$, so that $\mathcal{Z}_{g}$ shall have the expected dimension.

Using notation of Section 2.3, let $\mathcal{S}_{g, 1}^{*}:=\mathcal{S}_{g, 1} \cup A_{1,1}^{+} \cup A_{1,1}^{-}$be the partial compactification of $\mathcal{S}_{g, 1}$ such that the marked point on a nodal curve lies on an elliptic tail having even thetacharacteristic. Let $\left[C \cup_{q} E, p\right] \in \mathcal{W}$ be a general point and consider the class $\left[X, N, \alpha_{N}, p\right] \in \mathcal{S}_{g, 1}^{*}$, where $X=C \cup_{q^{\prime}} R \cup_{q^{\prime \prime}} E$ is the blow-up of $C \cup_{q} E$ at $q$, and $N$ is the line bundle such that $N_{\mid C}=\mathcal{O}_{C}\left((g-2) q^{\prime}\right), N_{\mid R}=\mathcal{O}_{R}(1)$ and $N_{\mid E}=\mathcal{O}_{E}\left((g-1)\left(p-q^{\prime \prime}\right)\right)$. Firstly, we want to show that the triple $\left[X, N, \alpha_{N}\right]$ lies in the boundary of the locus $\mathcal{S}_{g}^{r}$ of smooth spin curves possessing a theta characteristic with at least $r+1$ sections and the same parity as $r+1$. Namely,

Claim 4.2. $\left[X, N, \alpha_{N}\right] \in \overline{\mathcal{S}}_{g}^{r}$ and $\left[C \cup_{q} E\right] \in \overline{\mathcal{M}}_{g}^{r}$.

Proof of Claim 4.2. By Lemma 2.8, we only need to show that $\left[C, N_{\mid C}\right] \in \mathcal{S}_{g-1}$ lies on an irreducible component of $\mathcal{S}_{g-1}^{r}$ having codimension $\frac{r(r+1)}{2}$. Arguing as in the proof of Theorem 1.1, we achieve an inequality analogous to (4.1), that is

$$
\operatorname{dim} \mathcal{Z}_{g-1} \geq \operatorname{dim} \mathcal{P}^{\prime}+\operatorname{dim} \mathcal{Y}^{\prime}-\operatorname{dim} \mathcal{C}^{\left(g^{\prime}-1\right)},
$$

where $g^{\prime}=g-1$ is the genus of $C$ and $\mathcal{P}^{\prime}, \mathcal{Y}^{\prime}, \mathcal{C}^{\left(g^{\prime}-1\right)}$ are defined analogously. In particular, they satisfy $\operatorname{dim} \mathcal{P}^{\prime}=\operatorname{dim} U=3 g^{\prime}-2, \operatorname{dim} \mathcal{Y}^{\prime} \geq \operatorname{dim} U^{r}+r$ and $\operatorname{dim} \mathcal{C}^{\left(g^{\prime}-1\right)}=\operatorname{dim} U+g^{\prime}-1=4 g^{\prime}-3$. Since $\operatorname{dim} \mathcal{Z}_{g-1}=2 g^{\prime}-\frac{r(r-1)}{2}-1$ by assumption, we deduce that $\operatorname{dim} U^{r} \leq 3 g^{\prime}-2-\frac{r(r+1)}{2}$, and Theorem 2.7 assures that the latter is actually an equality. Thus any irreducible component of $\mathcal{S}_{g-1,1}^{r}$ containing $\mathcal{Z}_{g-1}$ has codimension $\frac{r(r+1)}{2}$ in $\mathcal{S}_{g-1,1}$. Hence the image of such a component under the forgetful map $\mathcal{S}_{g-1,1} \longrightarrow \mathcal{S}_{g-1}$ is an irreducible component of $\mathcal{S}_{g-1}^{r}$ having codimension $\frac{r(r+1)}{2}$ and passing through $\left[C, N_{\mid C}\right]$. Thus $\left[X, N, \alpha_{N}\right] \in \overline{\mathcal{S}}_{g}^{r}$ and $\left[C \cup_{q} E\right] \in \overline{\mathcal{M}}_{g}^{r}$ by Lemma 2.8 ,

Let $\left(\mathcal{X} \stackrel{\phi}{\longrightarrow} B, \xi, \alpha: \xi^{2} \longrightarrow \omega_{\phi}, B \stackrel{\rho}{\longrightarrow} \mathcal{X}\right)$ be the restriction to $\mathcal{S}_{g, 1}^{*}$ of a versal deformation family of $\left(X, N, \alpha_{N}, p\right)$ in $\overline{\mathcal{S}}_{g, 1}$, with $X_{b}:=\phi^{-1}(b), \xi_{b}:=\xi_{\mid X_{b}}, \alpha_{b}:=\alpha_{\mid \xi_{b}}$ and $p_{b}:=\rho(b)$. Hence 
$\left(X_{0}, \xi_{0}, \alpha_{0}, p_{0}\right)=\left(X, N, \alpha_{N}, p\right)$ for some $0 \in B$, and we have a commutative diagram of finite maps

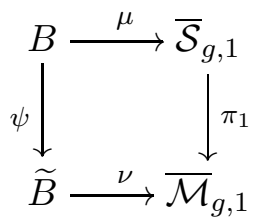

where $\widetilde{B}$ is a versal deformation space of $\left(C \cup_{q} E, p\right)$ in $\overline{\mathcal{M}}_{g, 1}$. Moreover, up to shrinking $B$, we can assume that $p_{b} \in X_{b} \backslash \operatorname{Sing}\left(X_{b}\right)$ for any $b \in B$.

Let $B^{r}:=\left\{b \in B \mid\left[X_{b}, \xi_{b}, \alpha_{b}\right] \in \overline{\mathcal{S}}_{g}^{r}\right\}$. Thanks to Claim 4.2 and Theorem 2.7, we have that $0 \in B^{r}$ and $\operatorname{dim} B^{r} \geq 3 g-2-\frac{r(r+1)}{2}$. Clearly, if $b \in B^{r}$ and $X_{b}$ is smooth, then $X_{b}$ coincides with its stable model $\widetilde{X}_{b}$ and the theta-characteristic $\xi_{b}$ gives a $\mathfrak{g}_{g-1}^{r}$ on $\widetilde{X}_{b}, L_{b}:=\left(\xi_{b}, V_{\widetilde{X}_{b}}\right)$, with $\xi_{b}^{2}=\omega_{\widetilde{X}_{b}}$ and $V_{\widetilde{X}_{b}} \subset H^{0}\left(X_{b}, \xi_{b}\right)$. On the other hand, if $X_{b}=C_{b} \cup_{q_{b}^{\prime}} R_{b} \cup_{q_{b}^{\prime \prime}} E_{b}$ for some $b \in B^{r}$, then Lemma 2.3 assures that the stable model $\widetilde{X}_{b}=C_{b} \cup_{q_{b}} E_{b} \in \overline{\mathcal{M}}_{g}^{r}$ admits a limit $\mathfrak{g}_{g-1}^{r}$, we denote by $L_{b}:=\left\{\left(\mathcal{L}_{C_{b}}, V_{C_{b}}\right)\left(\mathcal{L}_{E_{b}}, V_{E_{b}}\right)\right\}$, such that

$$
\mathcal{L}_{C_{b}}^{2} \cong \omega_{C_{b}}\left(2 q_{b}\right) \quad \text { and } \quad \mathcal{L}_{E_{b}}^{2} \cong \mathcal{O}_{E_{b}}\left((2 g-2) q_{b}\right) .
$$

In particular, conditions (4.3) are necessary to a limit $\mathfrak{g}_{2 g-2}^{g-1}\left\{\left(\mathcal{L}_{C_{b}}^{2}, W_{C_{b}}\right),\left(\mathcal{L}_{E_{b}}^{2}, W_{E_{b}}\right)\right\}$ for being a canonical limit linear series on $\widetilde{X}_{b}$ (see [7, p. 363]), and they provide locally closed conditions in the space of limit $\mathfrak{g}_{g-1}^{r}$ on $\widetilde{X}_{b}$. We note further that by Lemma 2.5, the curve $C \cup_{q} E$ admits a limit $\mathfrak{g}_{g-1}^{r}$ $L=\left\{\left(\mathcal{L}_{C}, V_{C}\right),\left(\mathcal{L}_{E}, V_{E}\right)\right\}$ satisfying conditions (4.3) and such that $a_{r}^{L}(p)=g-1, \mathcal{L}_{C}=\mathcal{O}_{C}((g-1) q)$ and $\mathcal{L}_{E}=\mathcal{O}_{E}((g-1) p)$. Following the recipe of [7, Section 3], we can thus base-change $B^{r}$ to get a family of triples $\left\{\left(\widetilde{X}_{b}, p_{b}, L_{b}\right) \mid b \in B^{r}\right\}$, where $\left(\widetilde{X}_{b}, p_{b}\right)$ is the stable pointed curve associated to $\left(X_{b}, p_{b}\right)$, the limit linear series $L_{b}$ is a limit $\mathfrak{g}_{g-1}^{r}$ as above, and $\left(\widetilde{X}_{0}, p_{0}, L_{0}\right)=\left(C \cup_{q} E, p, L\right)$.

Then we argue as in the proof of Theorem 1.1 and we consider the $(g-1)$-fold relative symmetric product $\widetilde{\mathcal{X}}^{(g-1)} \longrightarrow B^{r}$ of the family $\widetilde{\mathcal{X}} \longrightarrow B^{r}$, so that the fibre over each $b$ is the $(g-1)$ fold symmetric product $\widetilde{X}_{b}^{(g-1)}$ of the stable curve $\widetilde{X}_{b}$. We define two subvarieties of $\widetilde{\mathcal{X}}^{(g-1)}$ as $\widetilde{\mathcal{P}}:=\left\{p_{b}+\cdots+p_{b} \in \widetilde{X}_{b}^{(g-1)} \mid b \in B^{r}\right\}$, which is the image of the natural map $\widetilde{\rho}^{(g-1)}: B^{r} \longrightarrow \widetilde{\mathcal{X}}^{(g-1)}$ induced by $\rho$, and

$$
\widetilde{\mathcal{Y}}:=\left\{\begin{array}{l|l}
p_{1}+\cdots+p_{g-1} \in \widetilde{X}_{b}^{(g-1)} & \begin{array}{c}
b \in B^{r} \text { and } p_{1}+\cdots+p_{g-1}=(\sigma) \\
\text { for some global section } \sigma \text { of } L_{b}
\end{array}
\end{array}\right\},
$$

which is the locus of divisors $D_{b} \in \widetilde{X}_{b}^{(g-1)}$ where the sections of the limit linear series vanish. Consider the locus $B^{\prime}:=\left\{b \in B^{r} \mid p_{b}+\cdots+p_{b} \in \widetilde{\mathcal{P}} \cap \widetilde{\mathcal{Y}}\right\}$, and set $\mathcal{B}^{\prime}:=\left(\pi_{1} \circ \mu\right)\left(B^{\prime}\right) \subset \overline{\mathcal{M}}_{g, 1}$. We note that by construction of the versal deformation family in $\mathcal{S}_{g, 1}^{*}$, the locus $\mathcal{B}^{\prime}$ lies in $\mathcal{M}_{g, 1} \cup \Delta_{1,1}$, that is either $X_{b}$ is a smooth curve with $p_{b} \in X_{b}$, or $X_{b}=C_{b} \cup_{q_{b}} E_{b}$ is a nodal curve with $g\left(E_{b}\right)=1$ and $p_{b} \in E_{b}$. If $\widetilde{X}_{b}$ is a smooth curve, then $b \in B^{\prime}$ if and only if there exists a section $\sigma \in H^{0}\left(\widetilde{X}_{b}, \xi_{b}\right)$ such that $\operatorname{ord}_{p_{b}}(\sigma)=g-1$, i.e. $p_{b}$ is a subcanonical point of $\widetilde{X}_{b}$. Thus $\left[\widetilde{X}_{b}, p_{b}\right] \in \mathcal{G}_{g}^{r}$ and $\mathcal{B}^{\prime} \cap \mathcal{M}_{g, 1} \subset \mathcal{G}_{g}^{r}$. On the other hand, looking at nodal curves $\widetilde{X}_{b}=C_{b} \cup_{q_{b}} E_{b}$ with a marked point $p_{b} \in E_{b}$ on the elliptic tail, we have the following.

Claim 4.3. The locus $\mathcal{W} \subset \Delta_{1,1} \subset \overline{\mathcal{M}}_{g, 1}$ is an irreducible component of $\mathcal{B}^{\prime} \cap \Delta_{1,1}$. 
Proof of Claim 4.3. Let $D \subset B^{\prime}$ be an open disk centered at 0 . Our aim is to show that - up to shrinking $D-\left(\pi_{1} \circ \mu\right)(D) \subset \mathcal{W}$, that is $\left[\widetilde{X}_{b}, p_{b}\right] \in \mathcal{W}$ for any $b \in D$.

We note that $\left[\widetilde{X}_{b}=C_{b} \cup_{q_{b}} E_{b}, p_{b}\right] \in \mathcal{B}^{\prime} \cap \Delta_{1,1}$ if and only if there exists a global section $\sigma \in V_{E_{b}} \subset H^{0}\left(E_{b}, \mathcal{L}_{E_{b}}\right)$ such that $\operatorname{ord}_{p_{b}}(\sigma)=g-1$, i.e. $a_{r}^{L_{b}}\left(p_{b}\right)=g-1$. Thus Lemma 2.5 guarantees that for any $b \in D$, the point $q_{b} \in C_{b}$ is a subcanonical point with $h^{0}\left(C_{b},(g-2) q_{b}\right) \geq r$, and $p_{b}-q_{b} \in E_{b}$ is a torsion point of order $2 g-2$. In particular, the period of $p_{b}-q_{b}$ is exactly $2 g-2$, because this is the period of $p-q$ on the special fibre $E$.

So we want to show that $h^{0}\left(C_{b},(g-2) q_{b}\right) \equiv r+1(\bmod 2)$, which implies $h^{0}\left(C_{b},(g-2) q_{b}\right) \geq r+1$ also. We note that for any $b \in D$, we can endow the curve $X_{b}=C_{b} \cup_{q_{b}^{\prime}} R_{b} \cup_{q_{b}^{\prime \prime}} E_{b}$ with a line bundle $N_{b}$ such that $N_{b \mid C_{b}}:=\mathcal{O}_{C_{b}}\left((g-2) q_{b}^{\prime}\right), N_{b \mid E_{b}}:=\mathcal{O}_{E_{b}}\left((g-1)\left(p_{b}-q_{b}^{\prime \prime}\right)\right)$ and $N_{b \mid R_{b}}:=\mathcal{O}_{R_{b}}(1)$. Hence it is naturally defined a family of pointed spin curves $\left(X_{b}, N_{b}, \beta_{b}, p_{b}\right)$ parameterized over $D$, whose central fibre is $\left(X, N, \alpha_{N}, p\right)$. In particular $X=C \cup_{q^{\prime}} R \cup_{q^{\prime \prime}} E$, with $[C, q] \in \mathcal{G}_{g-1}^{r}$, $N_{\mid C}=\mathcal{O}_{C}\left((g-1) q^{\prime}\right)$ and $N_{\mid E}=\mathcal{O}_{E}\left((g-1)\left(p-q^{\prime \prime}\right)\right)$. Hence $h^{0}\left(C, N_{\mid C}\right) \equiv r+1(\bmod 2)$, and $h^{0}\left(E, N_{\mid E}\right)=0$ as the period of $p-q$ is exactly $2 g-2$. Since even and odd theta-characteristics do not mix (see Theorem 2.7), we deduce that $h^{0}\left(C_{b}, N_{b \mid C_{b}}\right) \equiv r+1(\bmod 2)$ as well. Thus $\left[C_{b}, q_{b}\right] \in \mathcal{G}_{g-1}^{r}$. Finally, as $\mathcal{Z}_{g-1} \subset \mathcal{G}_{g-1}^{r}$ is an irreducible component and $[C, q] \in \mathcal{Z}_{g-1}$ is a general point, we conclude that $\left[C_{b}, q_{b}\right] \in \mathcal{Z}_{g-1}$. Therefore $\left[\widetilde{X}_{b}=C_{b} \cup_{q_{b}} E_{b}, p_{b}\right] \in \mathcal{W}$, and hence $\left(\pi_{1} \circ \mu\right)(D) \subset \mathcal{W}$ as claimed.

We point out that $\operatorname{dim} \mathcal{B}^{\prime} \geq 2 g-\frac{r(r-1)}{2}-1$. Indeed

$$
\operatorname{dim} \mathcal{B}^{\prime}=\operatorname{dim} B^{\prime} \geq \operatorname{dim} \widetilde{\mathcal{P}}+\operatorname{dim} \widetilde{\mathcal{Y}}-\operatorname{dim} \widetilde{\mathcal{X}}^{\left(g^{\prime}-1\right)},
$$

where $\operatorname{dim} \widetilde{\mathcal{P}}=\operatorname{dim} B^{r} \geq 3 g-2-\frac{r(r+1)}{2}, \operatorname{dim} \widetilde{\mathcal{Y}}=\operatorname{dim} B^{r}+r$ and $\operatorname{dim} \widetilde{\mathcal{X}}^{(g-1)}=\operatorname{dim} B^{r}+g-1$. On the other hand, $\operatorname{dim} \mathcal{W}=\operatorname{dim} \mathcal{Z}_{g-1}+1=2 g-\frac{r(r-1)}{2}-2$. Therefore $\operatorname{dim} \mathcal{B}^{\prime}>\operatorname{dim} \mathcal{W}$, hence $\mathcal{B}^{\prime}$ must parameterize also smooth curves by Claim 4.3, that is $\mathcal{B}^{\prime} \cap \mathcal{M}_{g, 1} \subset \mathcal{G}_{g}^{r}$ is non-empty. Thus there exists an irreducible component $\mathcal{Z}_{g} \subset \mathcal{G}_{g}^{r}$ such that its closure $\overline{\mathcal{Z}}_{g}$ contains $\mathcal{W}$. In particular, $\overline{\mathcal{W}}$ is an irreducible component of $\overline{\mathcal{Z}}_{g} \cap \Delta_{1,1}$, and hence $\operatorname{dim} \mathcal{Z}_{g}=\operatorname{dim} \mathcal{W}+1=2 g-\frac{r(r-1)}{2}-1$.

We are now ready to prove Theorems 1.2 and 1.3 .

Proof of Theorem 1.2. Since $\mathcal{G}_{g}^{2} \subset \mathcal{G}_{g}^{\text {odd }}$ and the general point $[C, p] \in \mathcal{G}_{g}^{\text {odd }}$ is such that $h^{0}(C,(g-1) p)=1$ (see [4, Theorem 2.1]), we deduce $\operatorname{dim} \mathcal{G}_{g}^{2}<\mathcal{G}_{g}^{\text {odd }}=2 g-1$. Thus Theorem 1.1 assures that any non-empty irreducible component of $\mathcal{Z} \subset \mathcal{G}_{g}^{2}$ has dimension $\operatorname{dim} \mathcal{Z}=2 g-2$. To show that $\mathcal{G}_{g}^{2}$ is non-empty for any $g \geq 6$ we argue by induction on $g$. For $g=6$ the assertion follows from Example 3.1, whereas Theorem 4.1 leads to the assertion for any $g$.

Proof of Theorem 1.3. By arguing as above, Example 3.2 gives that $\mathcal{G}_{9}^{3}$ possesses an irreducible component of dimension $2 g-4$. Then by induction on $g$, the assertion follows from Theorem 4.1 .

We recall that given a pointed curve $[C, p] \in \mathcal{G}_{g}^{r}$, the smallest possible canonical ramification sequence $\alpha^{K_{C}}(p)$ at $p$ is

$$
\alpha:=(\underbrace{0, \ldots, 0}_{g-r-1}, \underbrace{r, \ldots, r}_{r}, g-1)
$$


and the corresponding sequence of Weierstrass gaps is $(1,2, \ldots, g-1-r, g, \ldots, g+r, 2 g-1)$ (see Section 2.1). Then, using upper semi-continuity, Theorem 4.1 gives a smoothing criterium for preserving the minimality of canonical ramification sequences, and hence the corresponding sequences of Weierstrass gaps. Namely,

Corollary 4.4. Under the assumption of Theorem 4.1, suppose that the general point $[C, q] \in \mathcal{Z}_{g-1}$ has canonical ramification sequence $\alpha^{K_{C}}(q)=(0, \ldots, 0, r, \ldots, r, g-2)$, as in 4.4). Then the general point of $\mathcal{Z}_{g}$ has canonical ramification sequence given by (4.4), as well.

Proof. Let $\left[C \cup_{q} E, p\right] \in \mathcal{W}$ as in (4.2), so that $\left[C \cup_{q} E, p\right] \in \overline{\mathcal{Z}}_{g} \subset \overline{\mathcal{G}}_{g}^{r}$. We then consider a family $(\mathcal{X} \stackrel{\phi}{\longrightarrow} T, T \stackrel{\rho}{\longrightarrow} \mathcal{X})$ of pointed curves such that $\left[X_{0}, p_{0}\right]=\left[C \cup_{q} E, p\right]$ is the central fiber, and $\left[X_{t}, p_{t}\right] \in \mathcal{Z}_{g}$ is a smooth pointed curve for any $t \neq 0$. The canonical bundles $\omega_{X_{t}} \cong \mathcal{O}_{X_{t}}\left((2 g-2) p_{t}\right)$ induce a limit $\mathfrak{g}_{2 g-2}^{g-1}$ on $C \cup_{q} E, H:=\left\{\left(\mathcal{H}_{C}, V_{C}\right),\left(\mathcal{H}_{E}, V_{E}\right)\right\}$, where

$$
\mathcal{H}_{C} \cong \omega_{C}(2 q) \cong \mathcal{O}_{C}((2 g-2) q) \text { and } \mathcal{H}_{E} \cong \omega_{E}((2 g-2) q) \cong \mathcal{O}_{E}((2 g-2) q)
$$

(see [7, p. 363]). Furthermore, $H_{C}:=\left(\mathcal{H}_{C}, V_{C}\right)$ is a complete linear series, that is $V_{C}=H^{0}\left(C, \mathcal{H}_{C}\right)$, because $\operatorname{dim} V_{C}=g$, the line bundle $\omega_{C}(q) \cong \mathcal{O}_{C}((2 g-3) q)$ has a base point at $q$, and $\mathcal{H}_{C}$ is basepoint free.

Letting $\alpha=\left(\alpha_{0}, \ldots, \alpha_{g-1}\right)$ be as in (4.4), we have that $\alpha^{H}(p) \geq \alpha^{\omega_{X_{t}}}\left(p_{t}\right) \geq \alpha$ by upper semicontinuity and minimality of $\alpha$ in $\mathcal{G}_{g}^{r}$ (see Remarks 2.1 and 2.2). In particular, setting $H_{E}:=$ $\left(\mathcal{H}_{E}, V_{E}\right)$, we have $\alpha^{H}(p)=\alpha^{H_{E}}(p)$ and the assertion shall follow from $\alpha^{H_{E}}(p)=\alpha$.

By the assumption on $\alpha^{K_{C}}(q)$ and the description of $H_{C}$, we have that its ramification sequence at $q \in C$ is $\alpha^{H_{C}}(q)=(0,1, \ldots, 1, r+1, \ldots r+1, g-1)$, that is $\alpha^{H_{C}}(q)=\left(\alpha_{0}, \alpha_{1}+1, \ldots, \alpha_{g-2}+\right.$ $\left.1, \alpha_{g-1}\right)$. Since $H$ is a limit $\mathfrak{g}_{2 g-2}^{g-1}$, compatibility conditions (2.2) give that the ramification sequence of $H_{E}$ at $q \in E$ is $\alpha^{H_{E}}(q)=\left(g-1-\alpha_{g-1}, g-2-\alpha_{g-2}, \ldots, g-2-\alpha_{1}, g-1-\alpha_{0}\right)$. Moreover, $\alpha_{i}^{H_{E}}(p)+\alpha_{g-1-i}^{H_{E}}(q) \leq g-1$ for any $0 \leq i \leq g-1$, as $H_{E}$ is a $\mathfrak{g}_{2 g-2}^{g-1}$ on $E$. Thus $\alpha_{0}^{H_{E}}(p)=\alpha_{0}$, $\alpha_{g-1}^{H_{E}}(p)=\alpha_{g-1}$, and $\alpha_{i} \leq \alpha_{i}^{H_{E}}(p) \leq \alpha_{i}+1$ for any $1 \leq i \leq g-2$. In particular, $g-2 \leq$ $\alpha_{i}^{H_{E}}(p)+\alpha_{g-1-i}^{H_{E}}(q) \leq g-1$. Then [8, Proposition 5.2] assures that for each $0 \leq i \leq g-1$, if $\alpha_{i}^{H_{E}}(p)+\alpha_{g-1-i}^{H_{E}}(q)=g-1$, then

$$
\left(\alpha_{i}^{H_{E}}(p)+i\right) p+\left(\alpha_{g-1-i}^{H_{E}}(q)+g-1-i\right) q \sim(2 g-2) p .
$$

Since the period of the torsion point $p-q \in E$ is exactly $2 g-2$, we conclude that $\alpha_{i}^{H_{E}}(p)+$ $\alpha_{g-1-i}^{H_{E}}(q)=g-1$ is satisfied only for $i=0$ and $i=g-1$. Thus $\alpha_{i}^{H_{E}}(p)=\alpha_{i}$ for any $0 \leq i \leq g-1$ and the assertion follows.

Finally, using the latter corollary and arguing as in Theorems 1.2 and 1.3 , we deduce the following.

Corollary 4.5. For any $g \geq 6$, there exists an irreducible component of $\mathcal{G}_{g}^{2}$ whose general point $[C, p]$ has canonical ramification sequence $\alpha^{K_{C}}(q)=(0, \ldots, 0,2,2, g-1)$.

Analogously, for any $g \geq 9$, there exists an irreducible component of $\mathcal{G}_{g}^{3}$ whose general point $[C, p]$ has canonical ramification sequence $\alpha^{K_{C}}(q)=(0, \ldots, 0,3,3,3, g-1)$.

In particular, the linear series $|(g-1) p|$ associated to such a general point is a base-point-free $\mathfrak{g}_{g-1}^{r}$ both when $r=2$ and $r=3$. 


\section{TRIPLY PERIODIC MINIMAL SURFACES}

In this section we deal with the application to periodic minimal surfaces. Initially, we recall the connection between periodic surfaces and theta-characteristics. Then we follow [20] and we state the main technical results leading to the proofs of Theorems 1.4 and 1.5.

We assume hereafter that $X$ is a compact connected Riemann surface of genus $g \geq 3$. In the following, we shall sometimes think $X$ as a complex algebraic curve, and we shall use the same notation of previous sections for the corresponding moduli spaces of Riemann surfaces.

We recall that a Riemann surface $X$ is periodic if admits a conformal minimal immersion $\iota: X \longrightarrow \mathbb{T}^{3}$ into a flat 3 -dimensional torus $\mathbb{T}^{3}=\mathbb{R}^{3} / \Lambda$. Using the classical Generalized Weierstrass Representation (see e.g. [15, 16, 2]), periodic surfaces may be characterized as follows (see [20, Section 0]). Given a Riemann surface $X$, we denote by $G_{\mathbb{Q}}:=G\left(3, H^{1}(X, \mathbb{Q})\right)$ and $G_{\mathbb{R}}:=G\left(3, H^{1}(X, \mathbb{R})\right)$ the Grassmannians of 3 -dimensional vector spaces in $H^{1}(X, \mathbb{Q})$ and $H^{1}(X, \mathbb{R})$, respectively, so that $G_{\mathbb{Q}} \subset G_{\mathbb{R}}$ is a dense inclusion. Then $X$ is a periodic surface if and only if there exists a theta-characteristic $\mathcal{L}$ on $X$ such that $h^{0}(X, \mathcal{L}) \geq 2$, the linear series $|\mathcal{L}|$ is base-point-free, and there exist two global sections $s_{0}, s_{1} \in H^{0}(X, \mathcal{L})$ not vanishing simultaneously such that the holomorphic 1-forms $\omega_{0}, \omega_{1}, \omega_{2} \in H^{0}\left(X, \omega_{X}\right)$ defined as

$$
\omega_{0}:=s_{0}^{2}-s_{1}^{2} \quad \omega_{1}:=i\left(s_{0}^{2}+s_{1}^{2}\right) \quad \omega_{2}:=2 s_{0} s_{1}
$$

satisfy $\operatorname{Span}\left(\operatorname{Re}\left(\omega_{0}\right), \operatorname{Re}\left(\omega_{1}\right), \operatorname{Re}\left(\omega_{2}\right)\right) \in G_{\mathbb{Q}}$. In this setting, the 4-tuple $\left(X, \mathcal{L}, s_{0}, s_{1}\right)$ is the spinor representation of the periodic surface $X$ (see e.g. [13]). Moreover, the set $\Lambda:=$ $\left\{\operatorname{Re}\left(\int_{\gamma} \omega_{0}, \int_{\gamma} \omega_{1}, \int_{\gamma} \omega_{2}\right) \in \mathbb{R}^{3} \mid \gamma \in H_{1}(X, \mathbb{Z})\right\}$ is a lattice of $\mathbb{R}^{3}$ of rank 3 and, after a translation, the conformal minimal immersion $\iota: X \longrightarrow \mathbb{R}^{3} / \Lambda$ is given by $\iota(p)=\operatorname{Re}\left(\int_{q}^{p} \omega_{1}, \int_{q}^{p} \omega_{2}, \int_{q}^{p} \omega_{3}\right)$, for some fixed point $q \in X$.

Thanks to the construction of [20, Section 2] and using notation of Section 2.3, we then introduce the moduli space $\mathcal{P}_{g}$ parameterizing 4-tuples $\left[X, \mathcal{L}, s_{0}, s_{1}\right]$, where $[X, \mathcal{L}] \in \mathcal{S}_{g}^{1} \cup \mathcal{S}_{g}^{2}$ is a smooth spin Riemann surface such that $h^{0}(X, \mathcal{L}) \geq 2$, and $s_{0}, s_{1} \in H^{0}(X, \mathcal{L})$ are two independent sections.

Given $\left[X, \mathcal{L}, s_{0}, s_{1}\right] \in \mathcal{P}_{g}$, it is possible to consider its versal deformation space $\left(\mathcal{X} \stackrel{\phi}{\longrightarrow} B, \mathcal{N}, \sigma_{0}, \sigma_{1}\right)$ in $\mathcal{P}_{g}$ (see [20, Section 3]), where $\mathcal{X} \stackrel{\phi}{\longrightarrow} B$ is a family of Riemann surfaces $X_{b}:=\phi^{-1}(b)$ of genus $g$, and $\mathcal{N}$ is a line bundle on $\mathcal{X}$ with sections $\sigma_{0}, \sigma_{1} \in H^{0}(\mathcal{X}, \mathcal{N})$ restricting on each fibre to a point $\left[X_{b}, \mathcal{N}_{b}, \sigma_{0, b}, \sigma_{1, b}\right] \in \mathcal{P}_{g}$. In particular, both the natural modular maps $\mu: B \longrightarrow \mathcal{P}_{g}$ and $\nu: B \longrightarrow \mathcal{M}_{g}$ are surjective with discrete fibres. We assume further that $B$ is simply connected and the family is complete, that is $\mu(B)$ contains an irreducible component of $\mathcal{P}_{g}$. Moreover, we denote by $B(\mathbb{Q}) \subset B$ sublocus of periodic surfaces.

The main technical result in [20] connects variations of a minimal immersion preserving the real periods of $\left(\operatorname{Re}\left(\omega_{0}\right), \operatorname{Re}\left(\omega_{1}\right), \operatorname{Re}\left(\omega_{2}\right)\right)$ up to the first order, and periodic Jacobi fields along such a minimal immersion. In particular, the variation of the period map at a point $\left[X, \mathcal{L}, s_{0}, s_{1}\right] \in \mathcal{P}_{g}$ is related by [20, Proposition 6.11] to the kernel of the Schrödinger operator $\Delta+|\nabla f|^{2}$ acting on real valued functions $u \in C^{\infty}(X)$, where $f: X \longrightarrow \mathbb{P}^{1} \cong S^{2} \subset \mathbb{R}^{3}$ is the holomorphic map defined by the linear system $\left|\operatorname{Span}\left(s_{0}, s_{1}\right)\right|, \Delta$ is the Laplace-Beltrami operator and $\nabla$ is the gradient (see [17]). Then [19, Theorem 2] and the Inverse Function Theorem lead to the following density result (see [20, Proposition 6.12 and Theorem 6.14]). 
Proposition 5.1. Let $\left[X, \mathcal{L}, s_{0}, s_{1}\right] \in \mathcal{P}_{g}$ be such that $s_{0}$ and $s_{1}$ do not have common zeroes, and $s_{1}$ vanishes at a single point. Let $B$ be a versal deformation space of $\left(X, \mathcal{L}, s_{0}, s_{1}\right)$ in $\mathcal{P}_{g}$. If $\operatorname{dim}_{\mathbb{R}} B=6 \mathrm{~g}$, then the locus $B(\mathbb{Q})$ is dense in $B$.

We can now prove Theorems 1.4 and 1.5 .

Proof of Theorem 1.4. The proof of assertions (i) and (iii) is actually included in [20, Section 6]. Then we assume $g \geq 6$ and we follow the very same argument to prove assertion (ii), which states that the locus $\mathcal{R}_{g}^{\text {odd }} \subset \mathcal{M}_{g}$ parameterizing odd periodic surfaces is dense in the locus $\mathcal{M}_{g}^{2}$ of surfaces admitting an odd theta-characteristic with at least three global sections. By Corollary 4.5, the locus $\mathcal{G}_{g}^{2} \subset \mathcal{M}_{g, 1}$ is non-empty, and its general point $[X, p]$ is such that $h^{0}(X,(g-1) p)=3$ and the linear series $|(g-1) p|$ is base-point-free. Setting $\mathcal{L}:=\mathcal{O}_{X}((g-1) p)$, there exist two sections $s_{0}, s_{1} \in H^{0}(X, \mathcal{L})$ without common zeroes, and we can assume that $s_{1}$ vanishes only at $p$ by definition of subcanonical point. Then we consider a complete versal simply connected deformation space $B$ of $\left(X, \mathcal{L}, s_{0}, s_{1}\right)$ in $\mathcal{P}_{g}$. We recall that any irreducible component of the locus of spin curves $\mathcal{S}_{g}^{2}$ has (complex) dimension $3 g-6$ (see e.g. [22, 10]). Moreover, any such a component passing through $[X, \mathcal{L}]$ has general point $[C, \xi]$ satisfying $h^{0}(C, \xi)=3$. By completeness, $\mu(B)$ contains an irreducible component of $\mathcal{P}_{g}$. Hence the real dimension of $B$ is $\operatorname{dim}_{\mathbb{R}}(B)=2\left(3 g-6+2 h^{0}(C, \xi)\right)=$ $6 g$. Thus Proposition 5.1 assures that $B(\mathbb{Q})$ is dense in $B$. By taking images under the modular map $\nu: B \longrightarrow \mathcal{M}_{g}$, we then deduce that $\mathcal{R}_{g}^{\text {odd }}$ is dense in $\mathcal{M}_{g}^{2}$.

Proof of Theorem 1.5. The assertions on even and hyperelliptic minimal surfaces are included in [20, Theorem 2]. Moreover, the proof of Theorem 1.4 assures that for any $g \geq 6$, there exists a point $\left[X, \mathcal{L}, s_{0}, s_{1}\right] \in \mathcal{P}_{g}$ satisfying $h^{0}(X, \mathcal{L})=3$ and the hypothesis of Proposition 5.1. Thus the assertion of Theorem 1.5] for odd minimal surfaces follows from [20, Section 6.19].

\section{ACKNOWLEDGEMENTS}

We are grateful to Gavril Farkas and Gabriele Mondello for helpful discussions.

\section{REFERENCES}

[1] E. Arbarello, M. Cornalba, P. A. Griffiths and J. Harris, Geometry of Algebraic Curves, Vol. I, Grundlehren der Mathematischen Wissenschaften [Fundamental Principles in Mathematical Sciences] 267, Springer-Verlag, New York, 1985.

[2] C. Arezzo and G. P. Pirola, On the existence of periodic minimal surfaces, J. Algebraic Geom. 8(4) (1999), $765-785$.

[3] L. Benzo, Components of moduli spaces of spin curves with the expected codimension, Math. Ann. 363(1) (2015), 385-392.

[4] E. M. Bullock, Subcanonical points on algebraic curves, Trans. Amer. Math. Soc. 365(1) (2013), 99-122.

[5] E. Colombo, On curves with a theta-characteristic whose space of sections has dimension 4, Math. Z. 215(4) (1994), 655-665.

[6] M. Cornalba, Moduli of curves and theta-characteristic, in Lectures on Riemann surfaces (Trieste, 1987), 560-589, World Sci. Publ., Teaneck, NY, 1989.

[7] D. Eisenbud and J. Harris, Limit linear series: basic theory, Invent. Math. 85(2) (1986), 337-371.

[8] D. Eisenbud and J. Harris, Existence, decomposition, and limits of certain Weierstrass points, Invent. Math. 87(3) (1987), 495-515.

[9] G. Farkas, Gaussian maps, Gieseker-Petri loci and large theta-characteristics, J. Reine Angew. Math. 581 (2005), 151-173.

[10] G. Farkas, Theta characteristics and their moduli, Milan J. Math. 80(1) (2012), 1-24. 
[11] P. A. Griffiths and J. Harris, Principles of Algebraic Geometry, Pure and Applied Mathematics, Wiley-Interscience [John Wiley \& Sons], New York, 1978.

[12] J. Harris, Theta-characteristics on algebraic curves, Trans. Amer. Math. Soc. 271(2) (1982), 611-638.

[13] R. Kusner and N. Schmitt, The spinor representation of minimal surfaces, e-print (1995), arXiv:dg-ga/9512003v1.

[14] M. Kontsevich and A. Zorich, Connected components of the moduli spaces of Abelian differentials with prescribed singularities, Invent. Math. 153(3) (2003), 631-678.

[15] H. B. Lawson Jr., Lectures on minimal submanifolds, Vol. I, Second edition, Mathematics Lecture Series 9, Publish or Perish, Wilmington, Del., 1980.

[16] W. H. Meeks III, The theory of triply periodic minimal surfaces, Indiana Univ. Math. J. 39(3) (1990), 877-936.

[17] S. Montiel and A. Ros, Schrödinger operators associated to a holomorphic map, Global differential geometry and global analysis (Berlin, 1990), 147-174, Lecture Notes in Math. 1481, Springer, Berlin, 1991.

[18] D. Mumford, Theta-characteristic on an algebraic curve, Ann. Sci. École Norm. Sup. (4) 4 (1971), 181-192.

[19] S. Nayatani, Morse index and Gauss map of complete minimal surfaces in Euclidean 3-space, Comment. Math. Helv. 68 (1993), 511-537.

[20] G. P. Pirola, The infinitesimal variation of the spin abelian differentials and periodic minimal surfaces, Comm. Anal. Geom. 6(3) (1998), 393-426.

[21] H. A. Schwarz, Gesammelte Mathematische Abhandlungen, Vol. I, Springer, Berlin, 1890.

[22] M. Teixidor i Bigas, Half-canonical series on algebraic curves, Trans. Amer. Math. Soc. 302(1) (1987), 99-115.

Dipartimento di Matematica e Fisica, Università degli Studi Roma Tre, Largo San Leonardo MuRIALDO 1, 00146 ROMA - ITALY

E-mail address: bastiane@mat.uniroma3.it

Dipartimento di Matematica, Università degli Studi di Pavia, via Ferrata 1, 27100 Pavia - Italy

E-mail address: gianpietro.pirola@unipv.it 